\title{
BER analysis on different channel based on OFDM and MIMO system
}

\author{
Prashant Verma ${ }^{1 *}$ and Ananad Khare ${ }^{2}$ \\ M.Tech Scholar, OIMT, Damoh, India ${ }^{1}$ \\ Assistant Professor, OIMT, Damoh, India ${ }^{2}$ \\ (C2016 ACCENTS
}

\begin{abstract}
Additive white Gaussian noise (AWGN) is utilized as a variation attribution for various procedures which may be random. It can be added with various noises to make the framework natural. Rayleigh fading is utilized to demonstrate the engendering model which can represent nonlinearity and scrambling. This major combination can be more successful when it can be tried with various adjustments in modulation. It is additionally compelling for the noise reduction. The data transmission with orthogonal frequency division multiplexing (OFDM) and multiple inputs and multiple outputs (MIMO) will be effective in the conjunction of AWGN and Rayleigh fading channel. This paper focuses on various attributes which can reduce the error rates with the help of bit error rate (BER).
\end{abstract}

\section{Keywords}

OFDM, MIMO, BER, AWGN, Rayleigh channel.

\section{Introduction}

OFDM is a productive system to calm the medium correspondence. OFDM is a rehash division multiplexing (FDM) plan used as an advanced multitransporter change strategy [1][2] so to speak OFDM is frequency division multiplexing of multi-bearers which are orthogonal to each other i.e. they are put precisely at the nulls in the regulation spectra of each other. This makes OFDM horrifyingly all the more convincing [3]. In OFDM information is detached into a couple parallel information streams or subchannels, one for every sub transport which are orthogonal to each other despite the way that they cover shockingly Each sub-transporter is controlled with a typical regulation arrangement, (for instance, QAM or PSK) at a low picture rate keeping up aggregate information rates like routine singletransporter change plots in the same transmission limit.

In today's situation MIMO is to an awesome degree obliging with the blend of OFDM structure. Manhandling the adaptability of MIMO frameworks recollecting the last goal to have high information rates is a particularly enchanting examination theme for future booking game plan organizes and their applications.

*Author for correspondence

62
Multiple input multiple output (MIMO) frameworks offer much more noteworthy channel limit over standard single-information single-yield structure.

As of different transmit Algorithms have been conveyed to encounter beyond what many would consider possible in the MIMO frameworks [4][5].Furthermore, in MIMO structures, coming about to selecting the get-together of clients with the at present most conspicuous achievable rates oversaw by a bundle scheduler in without come up short opening, we have to delegate them to the transmitter's radio wires in such a course, to the point that we can complete the best throughput in the framework. Assembled qualities techniques, for occurrence, space-time coding have gotten a huge amount of thought in perspective of their capacity to give higher unearthly gainfulness than routine single-information single-yield systems [6][7][8][9]. While applying this technique in a rehash particular channel, a space-time equalizer is required at the beneficiary to adjust for the impedance [10].

This multipath spread reasons discretionary time scattering, fixing, and stage improvement, known as clouding, in the got signal [11][12]. Clouding is accomplished by impediment between two or more sorts of the transmitted sign which associated at the power at to some degree unmistakable times [13]. DS-CDMA framework has the upsides of stretching out beyond what many would consider possible 
adjacent the resistance against staying [14][15][16]. In multi-client CDMA structures, multiple access interface (MAI) is seen as one of the standard wellsprings of execution degradation. Adaptable segregating systems have been enough used to level the direct and thusly reduce the MAI in the DSCDMA structure [17][18].

\section{Related work}

In 2011, Dubey et al. [19] proposed a Modernize SLM (MSLM) plan to decrease the PAPR by utilizing the mind boggling sign separate into certifiable and unconventional parts and autonomously orchestrate movement different true blue and non-existent piece of complex pennant then select smallest PAPR sign of genuine and eccentric and these are set. The multiplication show satisfies mind boggling PAPR, which is an in number contender for Future remote correspondence. In 2011, George A. Ropokis et al. [20] present a symptomatic bit error rate (BER) execution examination of different approaches under a common structure. All the more particularly, quite far and affiliation versatile courses of action are centred around, which separate in the way the choice on the transmitted picture is sent from the hand-off to the destination. The examination is done for a solitary hand-off $\mathrm{DaF}$ transmission convention and considers the photo choice bungles that might happen at the trade. Clear close structure definite BER expressions are found for every one of the three game plans, which are totally attested by diversions. In 2012, Mohamed Samir et al. [21] propose a change to the execution of a Direct Sequence Code Division Multiple Access (DS-CDMA) framework by using a versatile channel as a part of the area of unmistakable staying schedules. To battle the effect of such staying, the adaptable channel uses three versatile estimations which are the Variable Step-Size Affine Projection (VSS-APA) check, the Generalized Normalized Gradient Descent (GNGD) figuring, and the Generalized Square-Error-Regularized (GSER) NLMS calculation. As per the creators these checks have the benefits of smart meeting, low unsurprising state mean squared mistake and the capacity to redesign the bit error rate (BER) execution of the standard CDMA structure, in the locale of multi-way, different path, and unmistakable staying signs. Results show that the VSS-APA routs unmistakable estimations in the locale of surge sitting tight. While in the area of halfway band sticking with it NLMS adaptable channel gives the best execution. In 2014, Le et al. [22] demonstrate an exact piece botch rate estimation methodology for QPSK CO-OFDM transmission considering the probability thickness limit of the got QPSK pictures. Makers had been considered some known approaches, including data upheld and non-data screwed up vector size, they exhibit that the proposed strategy offers the most correct examination of the structure execution for both single channel and wavelength division multiplexing QPSK CO-OFDM transmission systems. In 2014, Zahed et al. [23] displayed a demonstrative approach to manage choose the impact of repeat balance, timing jitter and included adaptive white Gaussian noise (AWGN) on the bit botch rate (BER) execution of a Multi-carrier code division multiple access (MC-DS-CDMA) structure over a Rayleigh Fading Channel. The examination developed the PDF (probability thickness limit) at the gatherer considering joined effect of obscuring, timing jitter and Doppler repeat offset thus on with MRC plan. The expression for the prohibitive BER adjusted on a given timing bungle and obscuring is resolved and the ordinary BER is evaluated in the region of Multiple Access Interference (MAI) and Inter-Carrier Interference (ICI). The execution results are surveyed numerically with respect to SINR and BER considering system parameters like number of customers, number of sub-transporters. The result exhibits basic rot in SINR and BER execution as a result of obscuring close by the changes in parameters. In 2015, Kumar et al. [24] proposed a wavelet based SCFDMA for researching Bit Error Rate (BER) execution. Examination is did using particular wavelets and different conformity arranges under AWGN channel. This examination will exhibit that the diminishment in BER happens by using wavelet change as a piece of SCFDMA. Thusly wavelet based SCFDMA gives best BER execution over that of DFT based SCFDMA. In 2015, Jie et al.[25] proposed a MIMO-OFDM framework, which is the mix of MIMO and OFDM innovation for the high information transmission rate ease of use. They have recommended that MIMO-OFDM with STBC has great execution against Multi-way impacts and recurrence specific blurring, the BER and the coding intricacy are low. Their re-enactment model of MIMO-OFDM framework which depends on STBC is broke down on various transmission exhibitions under various channels.

\section{Problem formulation and analysis}

The attributes of remote sign changes as it goes from the transmitter radio wire to the beneficiary reception apparatus. These qualities rely on the separation between the two radio wires, the ways taken by the sign and the earth around the way. When all is said in 
done, the force profile of the got sign can be acquired by convolving the force profile of the transmitted sign with the drive reaction of the channel. Convolution in time area is proportionate to duplication in the recurrence space. In this manner, the transmitted sign $\mathrm{x}$, after engendering through the channel gets to be

$$
y(f)=H(f) x(f)+n(f)
$$

Channel response is denoted by $\mathrm{H}(\mathrm{f})$, and the noise is indicated by $n(f)$. The items situated around the way of the remote sign mirror the sign. Some of these reflected waves are likewise gotten at the collector. Since each of these reflected signs takes an alternate way, it has an alternate plentifulness and stage. Channel estimation can be performed in three ways. They are preparing based channel estimation, visually impaired channel estimation and semi visually impaired channel estimation. In preparing based channel estimation, known images are transmitted particularly to help the recipient's channel estimation calculations. Here, preparing images or pilot condition that are known from the earlier to the recipient, are multiplexed alongside the information stream for channel estimation. In a visually impaired channel-estimation system, the beneficiary must decide the channel without the guide of known images. The visually impaired channel estimation is completed by assessing the measurable data of the channel and certain properties of the transmitted signs.

Albeit higher-data transmission proficiency can be acquired in visually impaired procedures because of the bit error rate (BER). It is the figuring speaks to the rate of bit mistakes happening in an advanced information stream, for example, Internet or computerized phone signals. Bit blunders happen because of commotion or twisting in some piece of the circuit that causes a "1" to be gotten as a " 0 " and the other way around. The presence of bit blunders requires that mistake registering techniques be manufactured with correspondence frameworks to recognize such issues. Computerized transmissionstream quality can be assessed by contrasting the quantity of bits transmitted every second and the rate of those bits that must be retransmitted because of mistakes. Continuous observing of the BER remains a critical undertaking in keeping up top notch computerized correspondences.

This below Figure 1 to represent PSD (power spectrum density) or energy spectral 64 density (ESD), which is a positive real function of a frequency variable associated with a stationary stochastic process, or a deterministic function of time, which has dimensions of power per hertz $(\mathrm{Hz})$, or energy per hertz. So there is the need of reduction of BER with the channel mode representation.

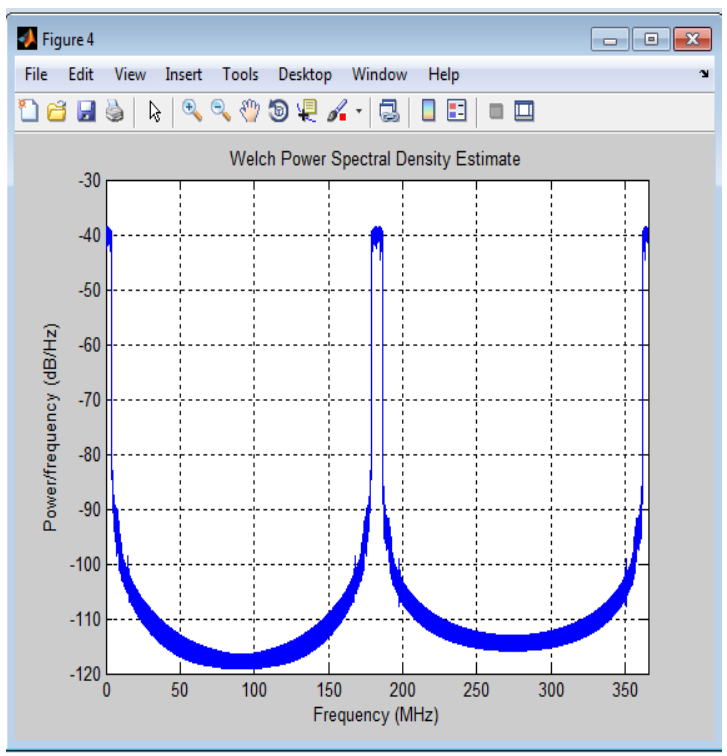

Figure 1 Power spectral density

The below Figure 2 represents spectral density of digital signal which is generated from discrete signal of our message signal.

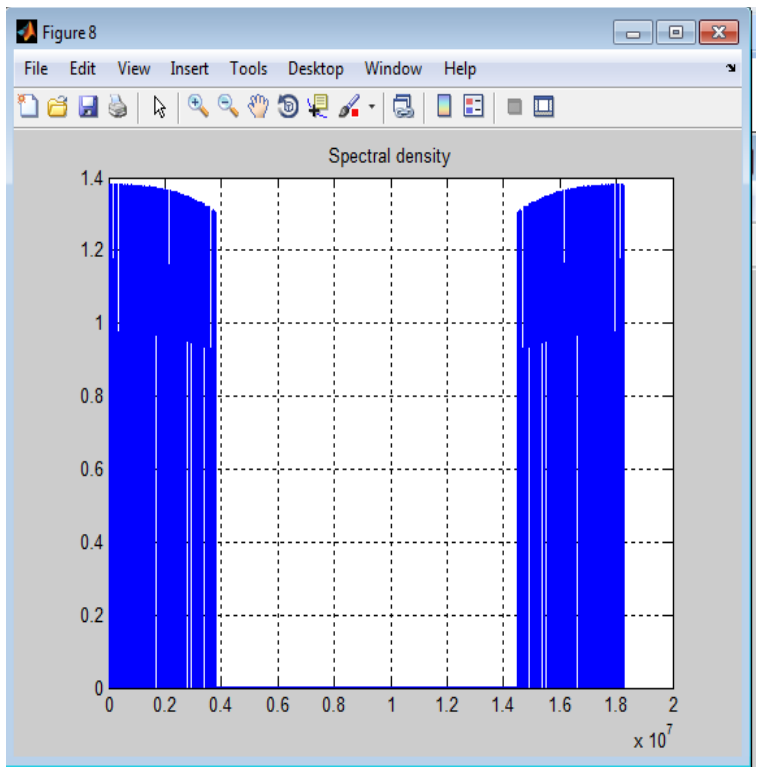

Figure 2 Power spectral density 
The below Figure 3 represents received signal in front of receiver side. It includes noise with addition of transmitted signal from the transmitter. We have to control the transmission distortion and temperature also be considered.

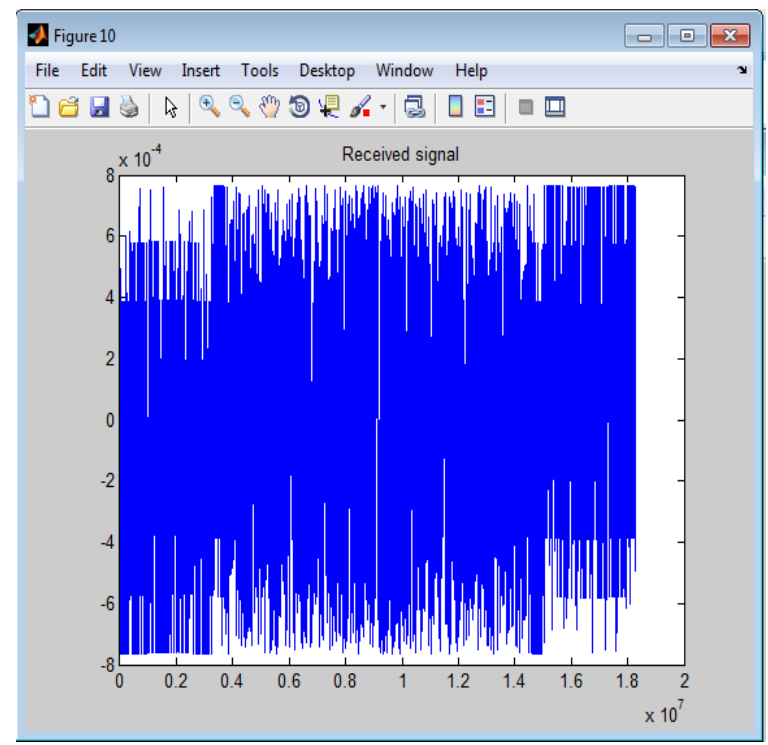

Figure 3 Received signal

We assume that the transmitter sends digital information by use of $\mathrm{M}$ signals waveforms $\{\mathrm{sm} \mathrm{g}$ $\{(\mathrm{t})=1,2, \ldots, \mathrm{M}\}$. Each waveform is transmitted within the symbol interval of duration $T$, i.e. $0 \leq t \leq T$. The channel is assumed to corrupt the signal by the addition of white Gaussian noise where $\mathrm{n}(\mathrm{t})$ denotes a sample function of AWGN process with power spectral density. Figure 4 is show Corrupted signal.

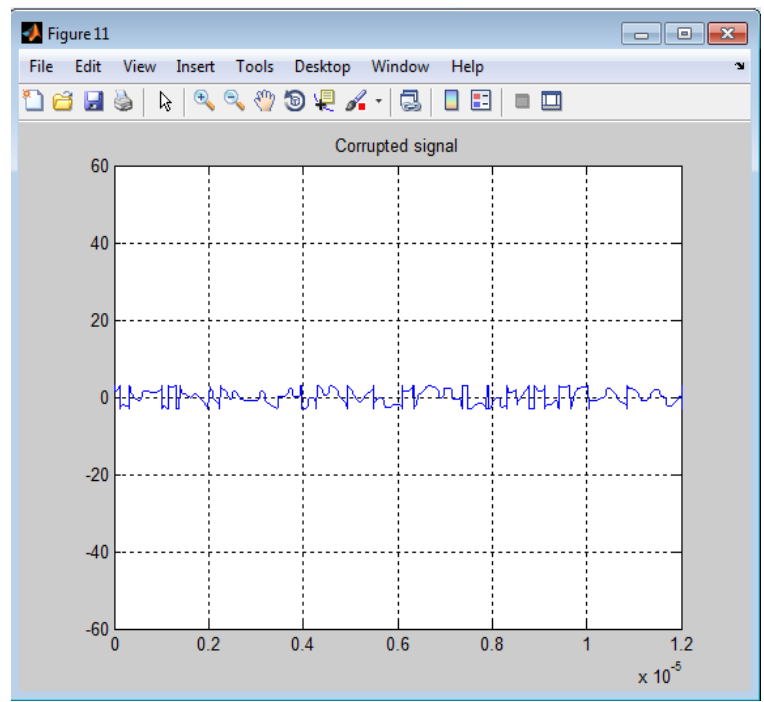

Figure 4 Corrupted signal

\section{Conclusion}

In view of the above investigation we can suggest that AWGN and Rayleigh channel with STBC can be productive in diminishing BER rates. It is more proficient on account of related conjunction of the transmitter and the beneficiary sign. It can be better with white timing jitter and opposite quick Fourier change too. The transmission fluctuation will be successful in the event that it is related with distinctive parameters.

\section{Acknowledgment}

None.

\section{Conflicts of interest}

The authors have no conflicts of interest to declare.

\section{References}

[1] Frederiksen FB, Prasad R. An overview of OFDM and related techniques towards development of future wireless multimedia communications. In radio and wireless conference 2002 (pp. 19-22). IEEE.

[2] Bingham JA. Multicarrier modulation for data transmission: an idea whose time has come. Communications Magazine, IEEE. 1990; 28(5):5-14.

[3] Wang Z, Ma X, Giannakis GB. OFDM or singlecarrier block transmissions? IEEE Transactions on Communications. 2004; 52(3):380-94.

[4] Trivedi S, Raeen MS, Pawar SS. BER analysis of MIMO-OFDM system using BPSK modulation scheme. International Journal of Advanced Computer Research. 2012; 2(5); 219-26.

[5] Xia P, Giannakis GB. Design and analysis of transmitbeam forming based on limited-rate feedback. IEEE Transactions on Signal Processing. 2006; 54(5):185363.

[6] Paramita S, Singh SS, Mohanta J. Time and frequency synchronization in OFDM system. International Journal of Advanced Computer Research. 2014; 4(16):856-65.

[7] Gupta V, Tiwari R. Performance analysis and simulation result of MC-CDMA for AWGN Channel and Rayleigh based on SNR/BER. International Journal of Advanced Computer Research. 2011; 1(1):13-17.

[8] Rathore M, Tanwar P. Reduction of co-channel interference in transmit/receive diversity (TRD) in MIMO System. International Journal of Advanced Computer Research.2012; 2(5):13-17.

[9] Gupta P, Singh UP, Richhariya V. Analysis and comparison of the 4-PSK and 8-PSK STTC over Rayleigh fading channels for determining performance. International Journal of Advanced Computer Research.2012; 2(5):142-9.

[10] Zhou S, Wang Z, Giannakis GB. Quantifying the power loss when transmit beam forming relies on finite-rate feedback. IEEE Transactions on Wireless Communications. 2005; 4(4):1948-57. 
[11] Tarokh V, Seshadri N, Calderbank AR. Space-time codes for high data rate wireless communication: Performance criterion and code construction. IEEE Transactions on Information Theory. 1998; 44(2):74465.

[12] Foschini GJ. Layered space-time architecture for wireless communication in a fading environment when using multi-element antennas. Bell Labs Technical Journal. 1996; 1(2):41-59.

[13] Anitha K, Sujatha BK. FPGA implementation of high throughput digital QPSK modulator using verilog HDL. International Journal of Advanced Computer Research. 2014; 4(14):217-22.

[14] Foschini GJ, Gans MJ. On limits of wireless communications in a fading environment when using multiple antennas. Wireless personal communications. 1998; 6(3):311-35.

[15] Telatar E. Capacity of Multi-antenna Gaussian Channels. European Transactions on Telecommunications. 1999; 10(6):585-95.

[16] Daksh JK, Mohan R, Sharma S. Performance analysis with space-time coding in MIMO-OFDM systems with multiple antennas. International Journal of Advanced Computer Research. 2013; 3(10):126-9.

[17] Choubey R, Mohan R, Sharma S. A survey of BER performance of generalized MC DS-CDMA system. International Journal of Advanced Computer Research. 2013; 3(2):130-3.

[18] Daksh JK, Mohan R, Sharma S. A survey of performance analysis in MIMO-OFDM systems. International Journal of Advanced Computer Research. 2013; 3(2):91-4.
[19] Dubey AK, Khandagre Y, Kushwaha GR, Hemnani K, Tiwari R, Shrivastava N. PAPR Reduction in OFDM by using modernize SLM technique. In recent trends in wireless and mobile networks 2011 (pp. 397-405). Springer Berlin Heidelberg.

[20] Ropokis GA, Rontogiannis AA, Berberidis K. BER performance analysis of cooperative $\mathrm{DaF}$ relay networks and a new optimal DaF strategy. IEEE Transactions on Wireless Communications. 2011; 10(4):1044-9.

[21] Samir M, Shaheen EM, Wahab AA. Performance analysis of DS-CDMA system using fast adaptive filtering under different jamming techniques. In international conference on engineering and technology (ICET), 2012 2012(pp. 1-6). IEEE.

[22] Le ST, Blow KJ, Mezentsev VK, Turitsyn SK. Bit error rate estimation methods for QPSK CO-OFDM transmission. Journal of Lightwave Technology. 2014; 32(17):2951-9.

[23] Zahed MI, Majumder SP. Performance analysis of MC-DS-CDMA in the presence of carrier frequency offset and timing jitter over Rayleigh Fading Channels. In international conference on computer and information technology (ICCIT) 2014 (pp. 1-5). IEEE.

[24] Kumar N. BER analysis in Wavelet based SC-FDMA for LTE uplink transmission. In international conference on advanced computing \& communication technologies (ACCT) 2015 (pp. 437-40). IEEE.

[25] Jie Z, Liang LI, Jin LI. Performance analysis of space time block code in MIMO-OFDM systems. In IEEE 3rd international conference on communication software and networks (ICCSN) 2011 (pp. 13-6). IEEE. 